\title{
Problemas, posibilidades y retos para la integración de los cultivos de frutos rojos en el paisaje del suroeste peninsular
}

\author{
Issues, potential and challenges for landscape \\ integration of berries' intensive crops in SW Iberian Peninsula \\ Morales Jiménez, J.'; Domingo-Santos, J.M.*; Pérez-Carral Lorenzo, C.'; \\ Corral-Pazos-de-Provens, E. ${ }^{1}$
}




\title{
Resumen
}

Los cultivos de frutos rojos o berries ocupan una gran superficie en el tercio sur de la provincia de Huelva. Se caracterizan por una agricultura tecnificada, intensiva, que se vale de invernaderos en túnel para la obtención de un producto de alto valor añadido, dado el alto precio de mercado que tienen los frutos rojos, así como por el empleo generado y las importantes industrias de procesado y servicios auxiliares. Este valor induce un uso intensivo del espacio, con grandes movimientos de tierras, rectificaciones y canalizaciones de arroyos, y ocupaciones de dominios públicos hidráulico, viario y otros. Así mismo, esta actividad genera importantes cantidades de residuos cuya manipulación es a menudo inadecuada. Este conjunto de circunstancias produce impactos paisajísticos significativos.

En el presente trabajo se identifican y describen los problemas señalados, y se plantean posibles estrategias de integración paisajística. Se definen tres tipos de medidas: i) aquellas que responden a situaciones ilegales que deben ser corregidas por los propietarios; ii) aquellas que identifiquen usos territoriales inadecuados no sancionables, cuya corrección puede desarrollarse instando al propietario a su resolución, incluso incentivándole; iii) medidas de mejora y creación de hábitats naturales y de la imagen visual general, cuyo desarrollo puede plantearse mediante incentivos y formación a los propietarios sobre las posibilidades de dar un uso terciario a sus explotaciones.

La integración paisajística de las explotaciones de frutos rojos es una necesidad importante para el desarrollo turístico de Huelva. Además, puede generar oportunidades para aquellos agricultores que se planteen generar espacios visitables que proyecten aún más hacia el exterior un producto y una denominación bien conocidos a escala mundial, pero que necesitan consolidar su imagen.

Palabras clave: berries, cultivos intensivos, gestión del paisaje, impacto paisajístico, restauración paisajística.

\begin{abstract}
Berry crops take a large area in the southern third of the province of Huelva. They feature a high-tech, intensive agriculture that uses tunnel greenhouses to obtain a product with high added value, in terms of price of the products and direct employment, as well as processing and ancillary industrial activities. This value drives to an intensive use of the land, with large earth-moving, straightening and channeling of water streams, and occupations of hydraulic, road and other public domains. Likewise, this activity generates significant amounts of waste whose handling is often inadequate. This set of circumstances produces significant landscape impacts. The present work identifies and describes the named problems, and proposes possible strategies for landscape restoration and integration. Three types of measures are defined: i) those that tackle illegal situations and thus need correction actions by the owners; ii) those that identify non-punishable irregular territorial uses whose correction can be developed by suggesting the owner to resolve them, subsidizing actions when possible; iii) actions for improvement and creation of natural habitats, as well as the enhancement of general visual quality, whose development can be raised through incentives and training the owners about the possibilities of giving a non-farming use to their farms. The integration of the berry landscapes is an important need for the tourist development of Huelva. In addition, it can arise as an opportunity for those farmers who consider hosting tourism to provide visitors with a unique experience that may promote their products abroad even more, and may also project a better image of this crops.
\end{abstract}

Keywords: berries, intensive crops, landscape management, landscaping impact, landscape restoration. 


\section{Introducción}

Desde que en los años 80 se empezaron a extender los cultivos de fresón en la provincia de Huelva y áreas limítrofes, el paisaje rural de las zonas donde se han ido implantando ha sufrido un profundo cambio. Las cubiertas vegetales previas, formadas por matorrales, pinares, eucaliptales y labores de secano, se han visto sustituidas por una producción intensiva, muy tecnificada, caracterizada por el cultivo bajo túneles de plástico transparente. El valor de las producciones es elevado, cada metro cuadrado de terreno cuenta, y esto ha desembocado en una desestructuración paisajística, debida a este aprovechamiento espacial tan intensivo.

En la última década, la diversificación de estos cultivos hacia otras especies y variedades de elevada rentabilidad (genéricamente denominados frutos rojos o berries) ha hecho que la demanda de territorio aumente y con ella el problema paisajístico. Así, los cultivos intensivos de berries ocupan una superficie que en 2018 ha superado las 15000 ha (Junta de Andalucía, 2018), en su mayor parte situada en zonas de alto valor ecológico y de demanda de uso público, dada su localización en las inmediaciones de la franja costera o dentro de algunos espacios protegidos, o ambas circunstancias, así como en las cercanías de muchos de los núcleos urbanos del sur de la provincia de Huelva.

Los frutos rojos representan hasta el 90\% del PIB de los municipios donde se ubican estos cultivos y "constituyen en sí mismos una marca" (Sánchez Haro, 2018).

El sector de los frutos rojos se caracteriza por disponer de asesoramiento técnico para el ejercicio de las labores del cultivo, sin embargo, los aspectos relacionados con el cuidado y la conservación del paisaje, la fauna y la vegetación, aún son poco conocidos y no asumidos de forma general por los agricultores (Villa et al., 2008).

El poder económico y social de este sector tan pujante, de esta marca, debe llevar a considerar un siguiente paso que sería la proyección de beneficios a otros sectores de actividad y a la sociedad en general. Esta proyección puede conseguirse, en gran parte, con la internalización de un importante conjunto de externalidades negativas generadas por la escasa conciencia de conservación ejercida por el sector hasta el momento.

El riesgo que se confronta, si no se tratan de solucionar los problemas ambientales, en general, y paisajísticos en concreto, puede tener un alcance muy importante, no solo para la población perjudicada por las externalidades negativas, si no también para los propios productores; constituirse en marca significa proyectar una imagen, adquirir un prestigio, fidelizar unos clientes que distribuyen los productos por los exigentes mercados europeos. La banalización y degradación de los espacios productivos (Zoido Naranjo, 2014) puede ser un claro motivo para que los clientes den la espalda a este sector y se vuelvan hacia otros países productores u otros productos.

En consecuencia, el sector debe asumir el doble papel que le asignan Villa et al. (2008) a los agricultores, por un lado, el papel de sustentador del sistema y, por otro, el de generador de paisajes agroambientales y, con ello, transformar un problema en una oportunidad de generar otros valores añadidos.

Este trabajo es una primera aproximación a la identificación y análisis de las 
principales situaciones problemáticas relacionadas con la integración visual de los cultivos de frutos rojos, situaciones que en su mayor parte repercuten sobre componentes de nuestro medio ambiente tan diversos como la geomorfología, la calidad de las aguas y ecosistemas asociados, la conectividad entre hábitats, etc., todos ellos constructores de la percepción del territorio que, como indica el Convenio de Florencia (Consejo de Europa, 2000), llamamos paisaje.

Para los principales problemas identificados se propondrán estrategias de mejora de la integración paisajística que resulten factibles, entendiendo por integración visual la conservación o introducción de elementos de mayor naturalidad que faciliten la comprensión del funcionamiento de los distintos sistemas territoriales (medio natural, redes de comunicación, red hidrográfica, etc.)

El objetivo final sería conseguir explotaciones modélicas cuya visita constituya un atractivo turístico y educativo per se, lo cual beneficiará al conjunto de la sociedad, tanto en la economía, como en la autoestima colectiva.

\section{Metodología}

Mediante recorridos de campo se ha realizado la observación de distintas casuísticas en las que se encontraban las explotaciones de frutos rojos, en relación a su calidad visual. En análisis especializados, hechos por la administración autonómica andaluza (Zoido Naranjo y Rodríguez Rodríguez, 2014), se ha vinculado la proliferación de estas explotaciones intensivas bajo plástico con la artificialización del paisaje, y se ha establecido la necesidad de su control estético por parte de las autoridades urbanísticas locales, principalmente en aquellas zonas de alta accesibilidad visual en las que pueden resultar compatibles usos turísticos. Resulta especialmente preocupante la simplificación paisajística que produce la ocupación casi total del territorio por los túneles de invernadero, los cuales borran cualquier seña de identidad que pueda permitir el aprecio de valores paisajísticos generales o particulares (Junta de Andalucía, 2012), así como una lectura de las relaciones anteriores entre el ser humano y su entorno.

Una vez constatado el problema, indicaremos que no se va realizar una valoración paisajística de las distintas escenas observadas basadas en métricas del paisaje o en la percepción pública, tal y como se describe en la bibliografía de referencia (p.e., Swanwick y Land Use Consultants, 2002; Muñoz Criado et al., 2012), dado que no se busca la protección de unas zonas o paisajes concretos, si no la reversión de la tendencia degradativa existente, mediante la implantación de buenas prácticas para la conservación paisajística y otras aportaciones a la conservación en general.

Las situaciones identificadas se han descrito en distintos aspectos, como el tipo de uso que se hace de los distintos espacios dentro de la explotación, ocupación del dominio público, uso de zonas de protección del dominio público, la presencia de desmontes del terreno, la colindancia con cursos de agua u otros impactos ecológicos y visuales.

La magnitud o incidencia territorial de cada uno de los tipos de situaciones problemáticas identificados se ha evaluado mediante técnicas de teledetección y 
SIG; en un segundo recorrido por el campo se contrastó que las situaciones impactantes identificadas mediante SIG tenían adecuado reflejo sobre el terreno. Si bien, estas mediciones no son objeto del presente trabajo, todas las situaciones para las que se proponen actuaciones correctoras tienen una importante ocurrencia. Algunas situaciones, como los grandes desmontes del terreno, aparecen principalmente en determinados términos municipales, mientras que otras situaciones como la degradación de los márgenes de cursos de agua y la ocupación del dominio público viario se encuentran generalizadas.

Para cada una de las situaciones descritas se establecen medidas correctoras orientadas hacia la problemática paisajística, pero que sin duda van a redundar en una mejora ecológica general. En concreto, se establecen tres escenarios sobre los que desarrollar medidas estratégicas de mejora:

1. Ocupaciones del Dominio Público (DP) cuya mejora paisajística debe asumir la Administración, previa recuperación del espacio ocupado, en colaboración con los propietarios colindantes, en la medida de lo posible. Este dominio público puede ser de índole diversa, principalmente DP viario, DP hidráulico, DP de vías pecuarias y montes de utilidad pública.

2. Usos territoriales inadecuados, cuya corrección puede beneficiar a la explotación en diversos aspectos, como la protección ante inundaciones o la seguridad de sus trabajadores, además de la calidad visual.

3. Presencia de condiciones favorables para la adopción de medidas de mejora y creación de hábitats naturales y de la imagen visual general de la explotación, con fines turísticos internalizables.

Finalmente, en la discusión se analiza la aplicabilidad de cada medida a los distintos tipos de problemas.

\section{Resultados}

En los recorridos de observación llevados a cabo se han observado un conjunto muy diversificado de problemas de índole paisajística. Cada explotación presenta sus propias circunstancias, tanto por las condiciones del terreno, como por las diferencias en la gestión, si bien, tratando de realizar una síntesis, se describen a continuación las principales situaciones "tipo".

\subsection{Grandes desmontes}

Se producen por la necesidad de allanar el terreno para el cultivo. Su aparición es muy frecuente, en casi todas las zonas en las que existen ondulaciones del terreno que forman pequeños cerros, que en la zona se denominan "cabezos". La destrucción de los cabezos significa una pérdida irreversible del relieve característico, además de fuertes perturbaciones en la dinámica de las aguas superficia- 
les y subterráneas. Además, cuando el cabezo no se desmonta totalmente, la explotación agrícola genera un corte del terreno que puede alcanzar desniveles importantes, de 6 a $8 \mathrm{~m}$ en paramentos casi verticales. Esta altura y verticalidad hace que la actuación sea muy visible, por lo que además de un efecto ecológico muy negativo, produce un impacto visual muy significativo (Figura 1-A).

Para la creación futura de explotaciones agrícolas estos grandes desmontes deben ser evitados, ya que existen alternativas menos impactantes como el aterrazado o abancalado.

En el caso de los desmontes ya existentes, la reducción de su impacto visual puede llevarse a cabo con el escalonamiento de los paramentos existentes en pequeñas plataformas con una anchura mínima de $1,5 \mathrm{~m}$ y desnivel de 1 a $1,5 \mathrm{~m}$ entre una y otra, según las características del terreno. Estas pequeñas terrazas se deben plantar con especies forestales que produzcan un efecto de pantalla sobre el desmonte (Figura 1-B). Además del efecto paisajístico, estas pantallas de especies forestales constituirán una barrera de retención de los materiales que la erosión hídrica pueda arrancar de los taludes, así como un refugio para las especies silvestres, incluida la fauna auxiliar, tan importante para la producción en agricultura ecológica y en agricultura integrada.

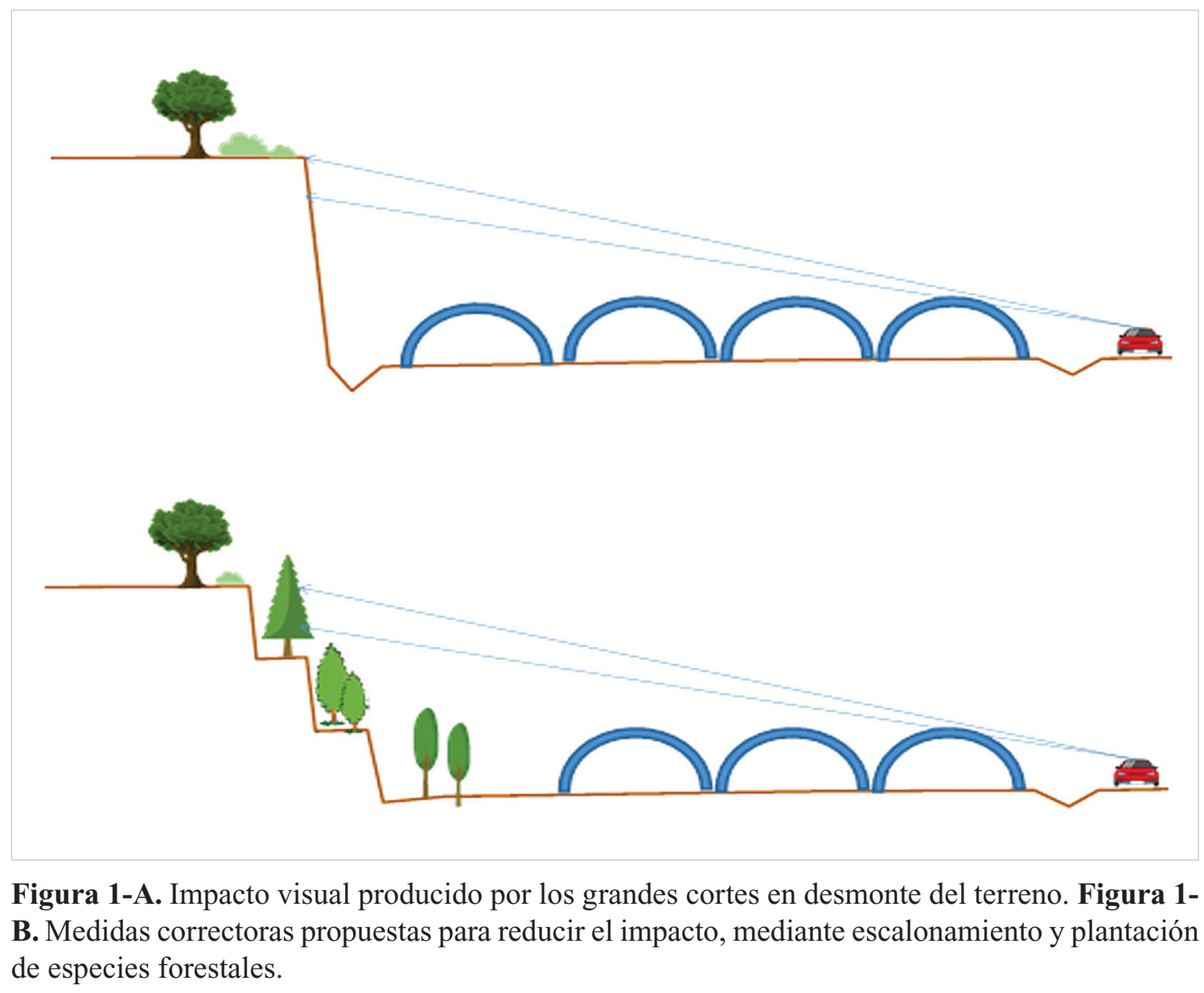




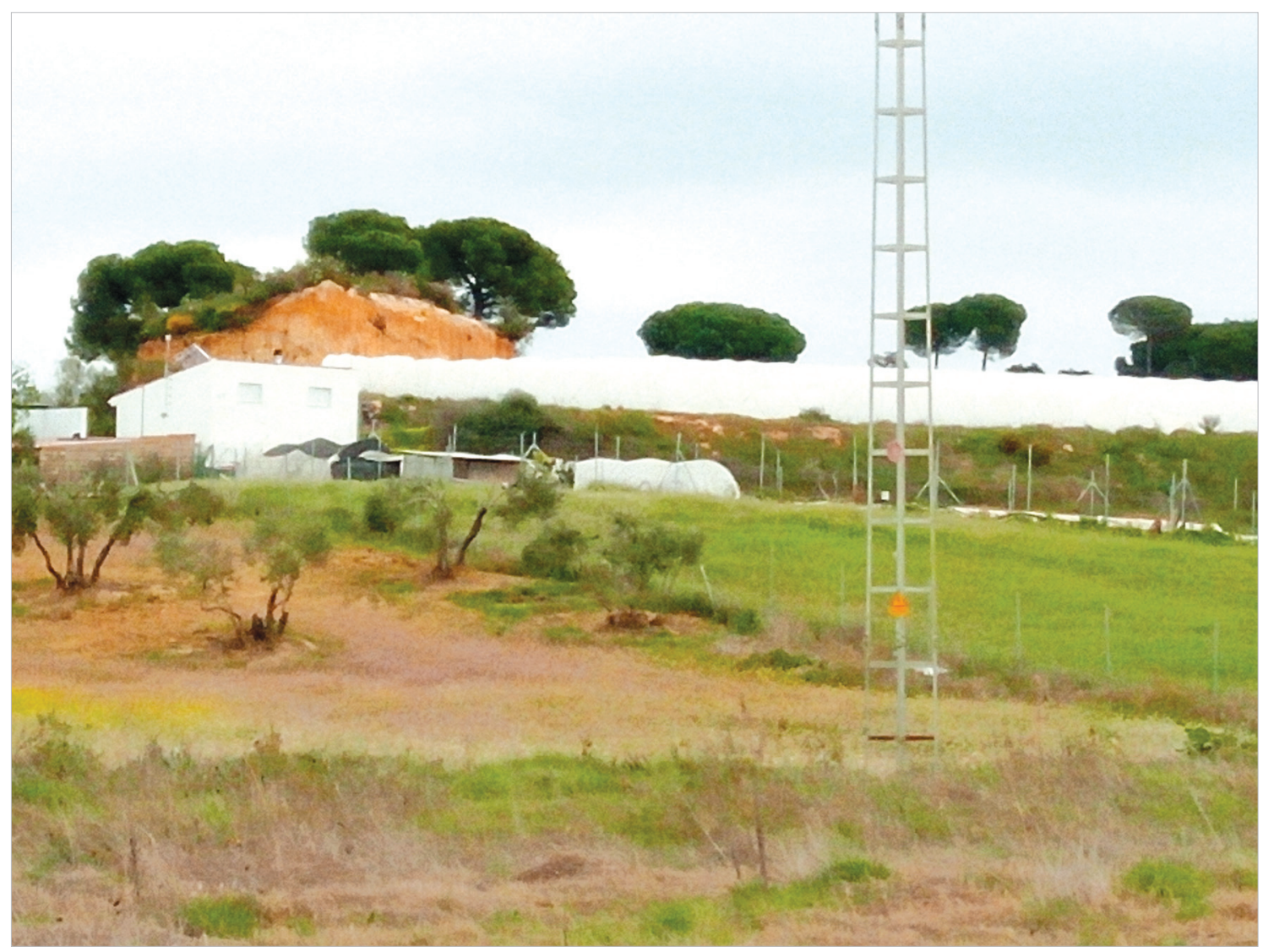

Foto 1. Generación de cortes verticales en el terreno con destrucción de la vegetación de pequeños cabezos y sus laderas.

Para la elección de las especies de plantación existe un manual específico (Guzmán Casado y Alonso Mielgo, 2008), al que se pueden añadir algunas especies locales no contempladas en ese documento. Las especies escogidas deben ser resistentes a las condiciones ambientales, de carácter autóctono, con crecimiento lo suficientemente rápido para generar pantallas visuales en un tiempo razonable, y con distintos desarrollos, para que ocupen varios pisos o estratos de vegetación; entre las especies recomendables estarían: pino piñonero (Pinus pinea), sabina mora (Juniperus phoenicea), alcornoque (Quercus suber), acebuche (Olea europaea var. sylvestris), algarrobo (Ceratonia siliqua), encina (Quercus ilex), adelfa (Nerium oleander), taray (Tamarix africana), arrayán (Mirtus communis), lentisco (Pistacea lentiscus) y torvisco (Daphne gnidium).

\subsection{Túneles de plástico y elementos acompañantes}

Los túneles de plástico son la base del sistema de producción intensiva de las berries. Por razones técnicas y económicas su uso se ha generalizado en detrimento de los microtúneles que son sistemas visualmente mucho más discretos, dado que apenas levantan $50 \mathrm{~cm}$ sobre el terreno, mientras que los túneles alcanzan unos 3 
m. La observación directa y cercana de los túneles de plástico genera una vista monótona y de baja calidad, especialmente cuando estos túneles ocupan grandes extensiones continuas y no existe ninguna barrera que amortigüe este efecto visual (Figura 2). La única barrera física suele ser un vallado de malla de simple torsión, que además de no ocultar o disimular los túneles, constituye un efecto visual negativo per se, reforzado por el contraste entre el color blanco de los plásticos y el gris metálico de la malla.

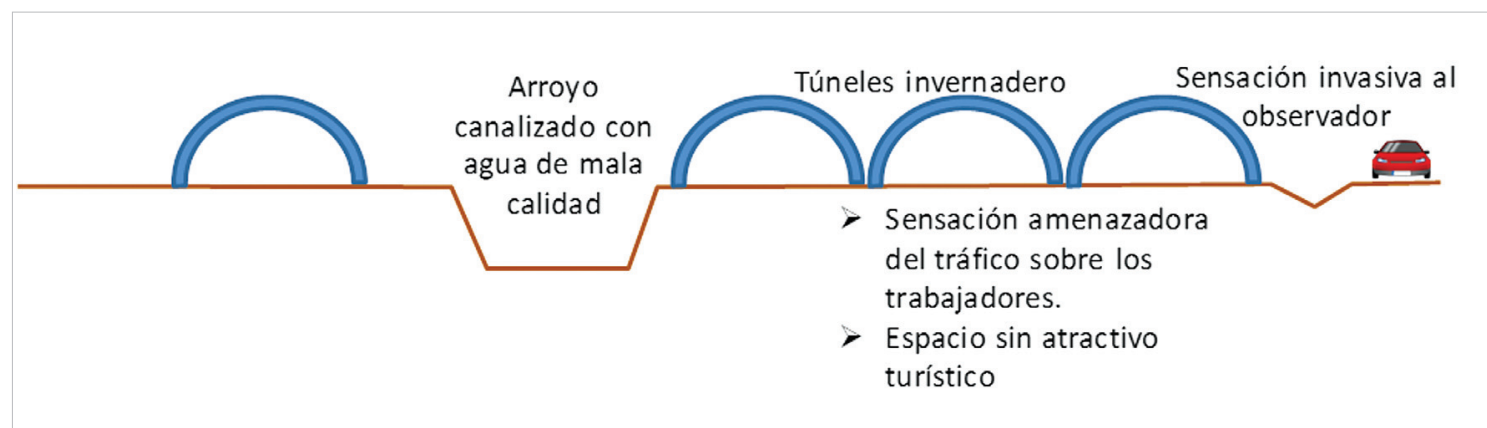

Figura 2. Situación actual de la mayor parte de los cultivos bajo túneles de plástico en relación a las carreteras (DP Viario y sus áreas de influencia) y las corrientes de agua (DP Hidráulico y áreas de influencia).

Como elementos acompañantes suelen aparecer casetas de bombas, almacenes de productos fitosanitarios, acopios de material, etc.

Como principales opciones para paliar los efectos visuales negativos de los túneles y elementos acompañantes se consideran, de forma no excluyente entre sí (Figura 3):

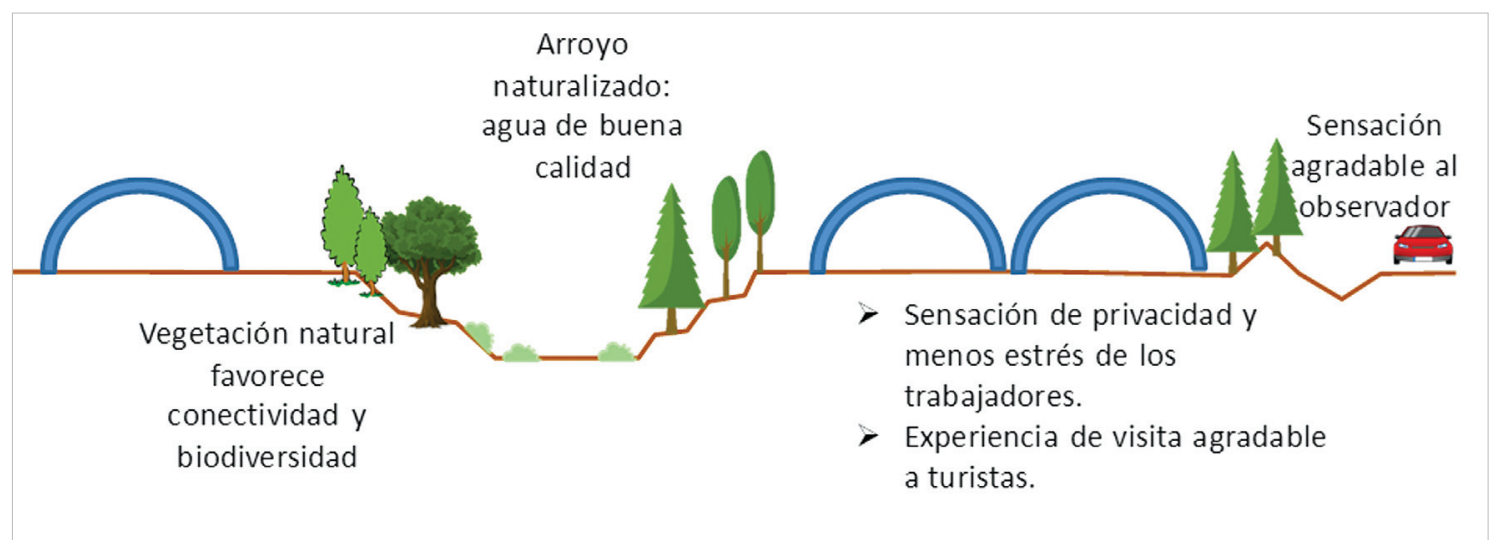

Figura 3. Propuestas de reducción de impactos visuales y ambientales, a la vez que se recuperan espacios de Dominio Público, cuando sea necesario.

- Apantallamientos: con vegetación, acompañada o no de pequeños montículos de terreno. Las pantallas vegetales, generan una sensación de mayor naturalidad, ya que rompen la monotonía de la vista de los plásticos, incluso si no alcanzan a 
ocultarlos totalmente. Para los trabajadores u otras personas que se encuentren en la explotación agrícola la pantalla provee una sensación de mayor sosiego, al reducir el ruido y la sensación amenazante del tráfico rodado, y dar una mayor privacidad. El efecto de apantallamiento y la calidad paisajística mejoran cuando la pantalla vegetal se coloca sobre terraplenes de tierra.

- Retranqueo de la explotación: el alejamiento en 10-15 m de los túneles de plástico, con respecto a la posición del observador mitiga el efecto visual, en especial si este espacio es ocupado por pantallas visuales y otros usos de menor impacto visual.

- Interrupción de la continuidad de los túneles: la plantación a intervalos de alineaciones de arbolado que superen en altura a los túneles ejerce un efecto mitigador muy eficaz. Para estas plantaciones pueden aprovecharse las redes de drenaje, así como la red viaria interna de la explotación. Además del efecto paisajístico positivo, el arbolado proporciona sombra y refugio a la fauna, y sus alineaciones contribuyen a la mejora de la conectividad ecológica.

Como especies recomendables para su uso en pantallas vegetales hay que tener en cuenta su capacidad de apantallamiento, así como su adaptación a las condiciones locales y el espacio disponible; algunas especies recomendables serían: Casuarina (Casuarina equistifolia), Grevillea (Grevillea robusta), Laurel (Laurus nobilis), Granado (Punica granatum), acebuche (Olea europaea var. sylvestris), Almez (Celtis australis), Higuera (Ficus carica). Como especies arbustivas se podrían utilizar las ya citadas para las zonas de talud: adelfa (Nerium oleander), taray (Tamarix africana), arrayán (Mirtus communis), lentisco (Pistacea lentiscus) y torvisco (Daphne gnidium).

Las zonas de retranqueo resultarían muy adecuadas para la plantación de olivos (Olea europaea), granados (Punica granatum), naranjos (Citrus $x$ sinensis) u otros árboles frutales de desarrollo moderado.

En las alineaciones o bosquetes de arbolado destinados a romper la continuidad de la superficie de plástico resultarían adecuadas especies autóctonas con un cierto desarrollo en altura como pino piñonero (Pinus pinea), sabina mora (Juniperus phoenicea), alcornoque (Quercus suber), acebuche (Olea europaea var. sylvestris), algarrobo (Ceratonia siliqua), o encina (Quercus ilex).

\subsection{Invasión del Dominio Público Hidráulico}

Cuando un arroyo u otro cauce de mayor magnitud desarrolla su recorrido por fincas de distintos propietarios, su carácter es de bien o dominio público y queda englobado dentro del Dominio Público Hidráulico (DPH).

Pertenezca o no al DPH, la red de drenaje ocupa espacio y genera riesgos de inundación, por lo que una acción muy frecuente en las zonas agrícolas es la destrucción o degradación de humedales, arroyos u otros elementos de la red hidrológica. La acción más frecuente es la rectificación y canalización de esta red hídrica, para que ocupe el menor terreno posible y evacúe rápidamente las escorrentí- 
as. La canalización de estas corrientes de agua supone una pérdida de biodiversidad que queda directamente reflejada en la degradación de los paisajes. Además, supone un riesgo muy importante ante sucesos de avenidas extraordinarias, pues pueden generarse importantes daños en los espacios "ganados al cauce" y en las propiedades que se encuentren aguas abajo de la rectificación.

Como principales acciones correctoras se proponen (Figura 3):

- Identificación de las redes de drenaje principal en las explotaciones agrarias y su estado.

- Ensanche escalonado de los encauzamientos: este ensanche mejora la capacidad de drenaje y hace más improbable las posibilidades de inundación de la explotación agrícola y de las que se encuentren aguas abajo y aguas arriba. Las dimensiones del ensanche deben verse determinadas por cálculo hidrológico, si bien, al menos, sobre el lecho del cauce se deben levantar escalones de 1-1,5 m de ancho, por cada 0,5-1 m de altura. Cuando se trate de DPH la anchura de la actuación afectará al menos a los límites del Dominio.

- Plantación de las márgenes de cauces y elementos principales de las redes de drenaje. La plantación de al menos la última línea de terrazas del cauce (cuando este aterrazamiento se ejecute), así como de las márgenes, genera un efecto paisajístico de rotura de la continuidad de los plásticos, además de la mejora ecológica.

Como especies recomendables para su uso en el primer escalón de restauración tendríamos especies de vegetación de ribera como: fresno (Fraxinus angustifolia), aliso (Alnus glutinosa), sauces (Salix sp.); como especies arbustivas se pueden utilizar: adelfa (Nerium oleander), taray (Tamarix africana), o madroño (Arbutus unedo).

Para la orla de vegetación más separada del agua se pueden utilizar las ya citadas para taludes, alineaciones y bosquetes: pino piñonero (Pinus pinea), sabina mora (Juniperus phoenicea), alcornoque (Quercus suber), acebuche (Olea europaea var. sylvestris), algarrobo (Ceratonia siliqua), encina (Quercus ilex), granado (Punica granatum), almez (Celtis australis), higuera (Ficus carica). Como especies arbustivas se podrían utilizar las ya citadas para las zonas de talud: arrayán (Mirtus communis), lentisco (Pistacea lentiscus) y torvisco (Daphne gnidium).

Dada la enorme variedad de arbustos autóctonos que vegetan en esta área, sería muy conveniente investigar la interacción que cada especie puede presentar con la fauna auxiliar y la fauna predadora de los cultivos de berries, con el fin de ampliar el número de especies utilizado y conseguir así pequeños hábitats de mayor riqueza.

\subsection{Invasión del Dominio Público Viario (DPV)}

La Ley 8/2001 de Carreteras, de la Junta de Andalucía, establece la obligatoriedad de llevar a cabo un proyecto de restauración paisajística, que puede acom- 


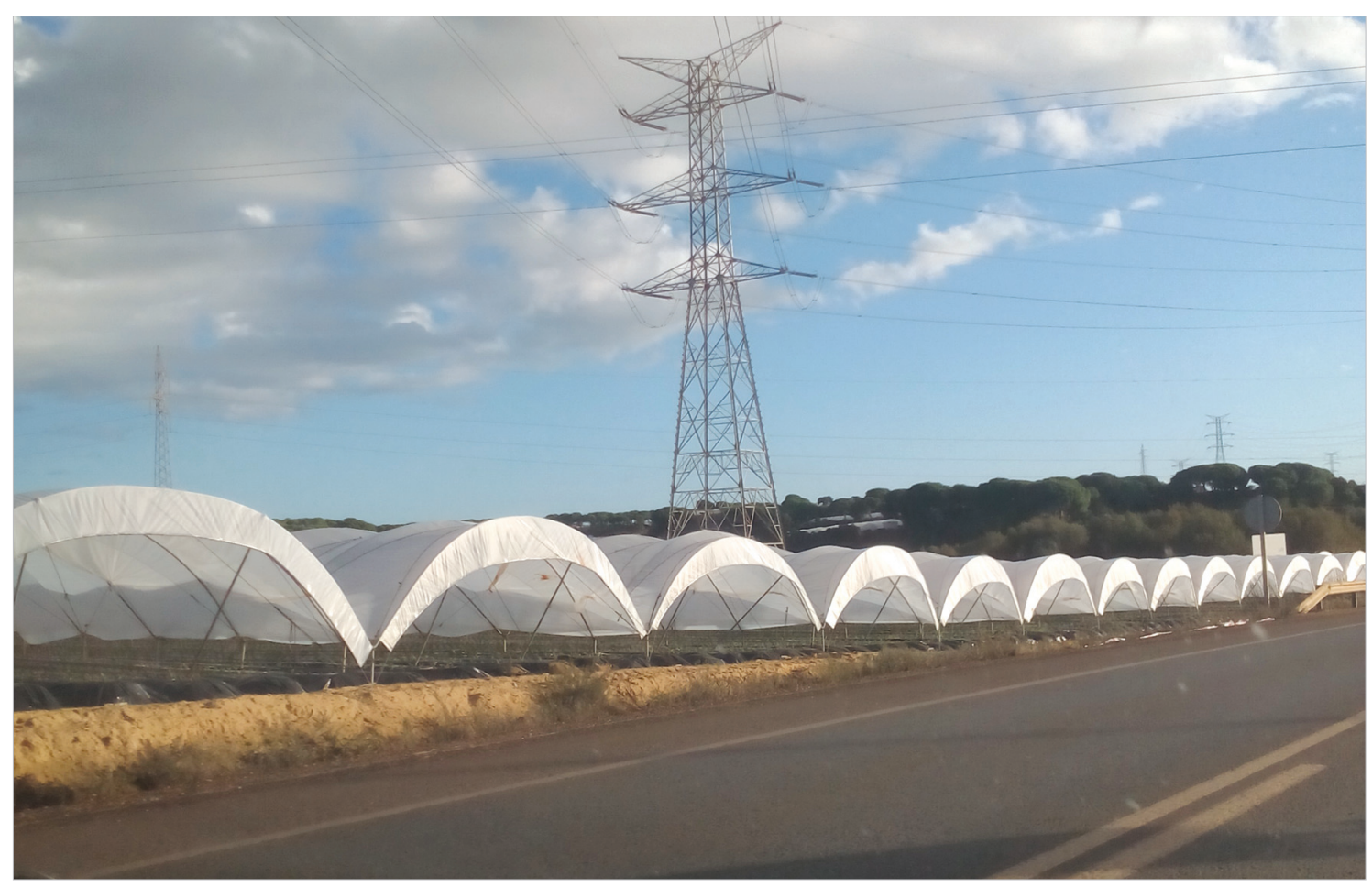

Foto 2. Ocupación del Dominio Público Viario por los cultivos de berries y sus servicios auxiliares.

pañar al proyecto de construcción (o reforma) de la carretera, o ser redactado de forma independiente. Se establece, además, que este proyecto de restauración, que debe perseguir la integración paisajística de la carretera en su entorno, pueda trasponer los límites del Dominio público adyacente y utilizar la franja de Servidumbre legal. En la Zona de Afección establece limitaciones a la construcción o cualquier otra acción que pueda afectar a la integración paisajística.

La invasión del Dominio público por parte de las explotaciones agrarias es un hecho bastante frecuente, y también lo es el uso de la zona de Servidumbre legal para la colocación de los túneles invernadero.

Como acciones correctoras se plantean:

- La identificación de la ocupación del DPV por parte de las explotaciones agrarias.

- Articulación de convenios para redactar proyectos de integración paisajística en el DPV y la zona de Servidumbre legal; en éstos, la parte de actuaciones del proyecto sobre DPV debe ser sufragada por la Administración y la parte de Servidumbre legal por el particular, que podrá acogerse a líneas de ayuda, siguiendo los consejos en relación a las especies más aptas para la formación de setos (p.e. Guzmán Casado y Alonso Mielgo, 2008).

- Las principales acciones restauradoras serán las barreras de vegetación, con otros posibles elementos como terraplenes elevados, según ya se ha descri- 


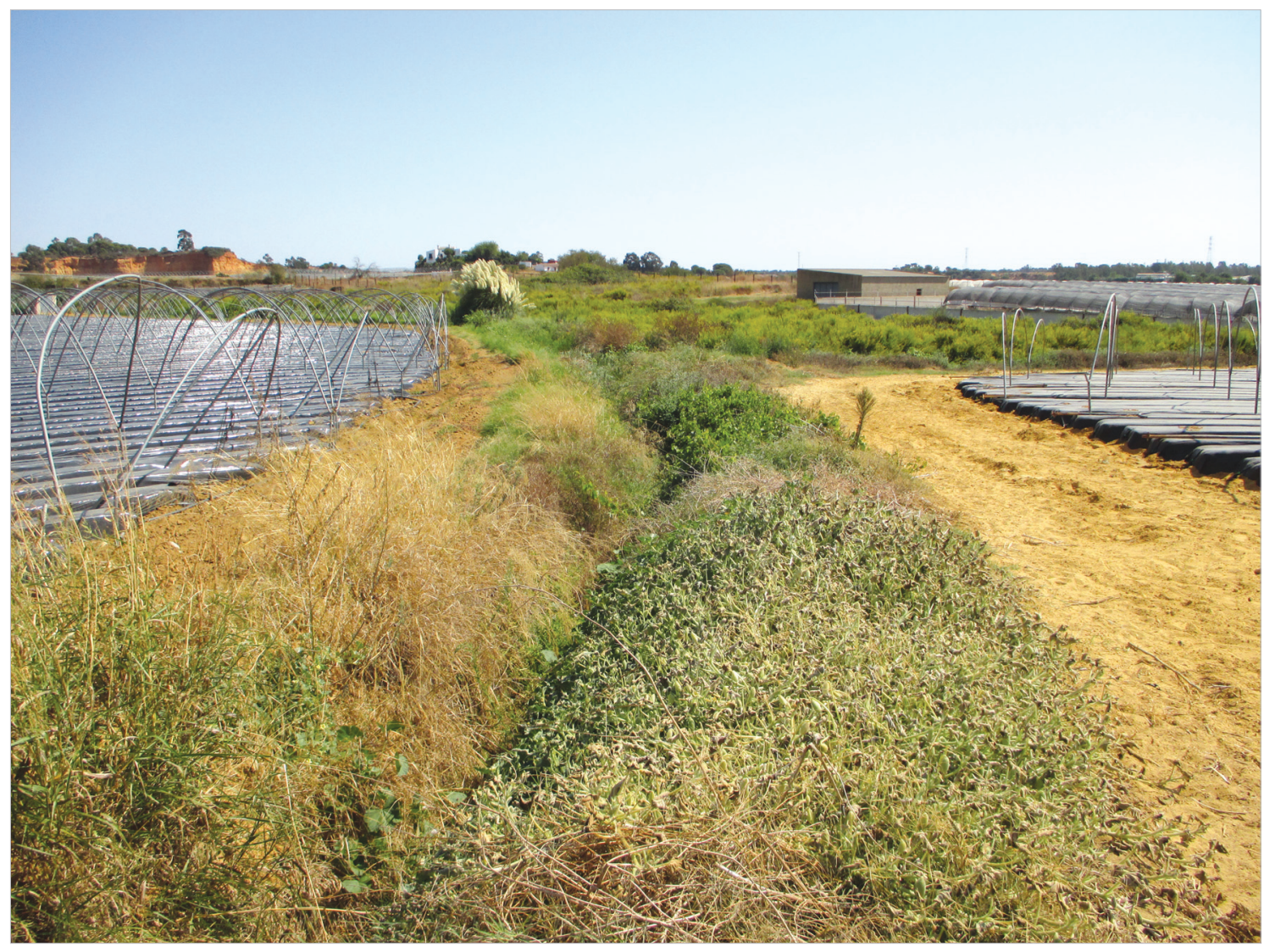

Foto 3. Degradación de un arroyo por la eliminación de su vegetación natural y la proliferación de matorrales de degradación, de escaso valor.

to anteriormente. Esta acción se puede complementar con la retirada de algún módulo de túnel cuando se considere necesaria. Otra opción es utilizar toda o parte de la zona de afección para otros usos agrícolas de tipo tradicional, con un impacto visual positivo, como viña, olivar, agrios, $u$ otros frutales, lo que haría innecesario el uso de pantallas vegetales densas.

\subsection{Otros problemas}

Existen otros problemas diversos para los cuales se deben buscar soluciones ad hoc. En ocasiones estos problemas no son más que conductas sancionables o al menos reprobables, cuya corrección o solución se debe solicitar al agricultor por las diversas vías administrativas, entre estas se pueden destacar:

- Acumulación de restos de plásticos en zonas visibles, en ocasiones incluso en zonas forestales públicas. Además de plásticos de invernadero, pueden ser procedentes de envases de productos fitosanitarios, tubería de riego y otros.

- Presencia basura de origen doméstico, generada por los trabajadores que almuerzan (o incluso pernoctan) en la finca. 


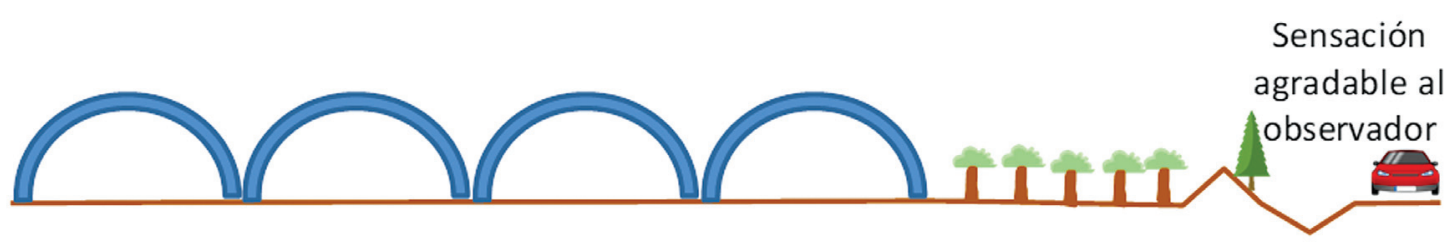

Figura 4. Retranqueo de túneles de invernadero; el espacio que ocupaban no deja de ser productivo, ya que se utilizaría para otros cultivos de mejor integración paisajística, por carecer de estructuras artificiales y cubiertas plásticas.

- Ocupación del monte u otros dominios públicos con acopios de materiales.

- Hormigonado de grandes superficies para proteger el terreno de la erosión por escorrentía. En ocasiones el hormigón es sustituido por una cubierta plástica. En ambos casos se generan escorrentías violentas en la zona de desagüe de la finca.

- Contaminación y eutrofización de arroyos por el arrastre de fertilizantes.

\section{Discusión}

Más allá de todas las posibles soluciones enunciadas, queda claro que el problema subyacente es el alto valor añadido que tiene la superficie de producción de berries; renunciar a una fracción de este espacio productivo, por pequeña que sea, probablemente supone una preocupación mayor que tener que afrontar los costes de ejecución de acciones restauradoras, que, en general, serán poco significativos $\mathrm{y}$, además, subvencionables (Junta de Andalucía, 2017).

Lógicamente, renunciar a una pequeña parte del espacio productivo no representa el mismo impacto económico en una explotación grande que en una intermedia o una pequeña. Las compensaciones futuras tampoco son las mismas, por lo que las exigencias de actuación deben acomodarse a la dimensión económica de la explotación.

Son precisamente las explotaciones de mayor tamaño las que pueden beneficiarse de un uso turístico de estos espacios, en los que se producen berries que surten los mercados internacionales más exigentes; esto debe pasar por la dotación de instalaciones que ofrezcan un espacio armonioso, con edificaciones tradicionales tipo cortijo, patios andaluces, jardines, agua, espacios de exposición y degustación, etc. Por otro lado, los terrenos de cultivo no deben perder su funcionalidad, pero deben resultar de visita agradable, integrados con su entorno, libres de basura, de residuos, de olores desagradables, o de los insectos que proliferan en esas condiciones. La visita debe representar una experiencia cercana a la actividad agrícola, pero asegurándose que se proyecta una imagen de limpieza y naturaleza, un descubrimiento de sensaciones visuales, olfativas y gustativas.

Las estrategias de acción para la consecución de las medidas propuestas tienen tres posibles líneas de desarrollo: 
1. Medidas de mejora de espacios de Dominio Público, destinadas principalmente a la defensa de éste y a su revalorización ambiental. Estas medidas deben ser puestas en marcha directamente por la Administración, previa información a los colindantes; en ocasiones, si se pretende que las medidas afecten a las zonas de servidumbre, se debe alcanzar un acuerdo con su propietario, para el tipo de acciones, así como para su financiación, que puede ser subvencionada.

2. Medidas de policía destinadas a corregir vulneraciones de la normativa por parte de los agricultores. Deben seguir los procedimientos administrativos que usen vías de acercamiento negociado, antes de pasar a las medidas coercitivas.

3. Medidas voluntarias de mejora paisajística de las explotaciones. Aquellas que constituyan una apuesta clara de los empresarios agrícolas por darle una nueva dimensión de uso público a su negocio.

\section{Conclusiones}

Se han identificado varios problemas de índole visual-paisajístico en las plantaciones de frutos rojos (berries) de la provincia de Huelva, casi todos ellos con otro tipo de implicaciones para la conservación de la biodiversidad, la calidad de las aguas, el riesgo de incendios, etc.

La solución a la mayor parte de los problemas pasa por el establecimiento de zonas de transición entre las zonas dedicadas a la agricultura intensiva y los espacios de uso público desde los que se observan estos cultivos. Estas zonas de transición deben ofrecer una adaptación de la topografía y un apantallamiento vegetal. Las soluciones ofrecidas se consideran eficaces, sencillas y poco costosas de implementar.

El mayor problema para el desarrollo de las zonas de transición es la pérdida de una parte del terreno productivo; esta pérdida será por regla general muy pequeña, si bien en explotaciones de tamaño reducido sí podría ser significativa.

La mejora paisajística integral de una explotación de berries puede facilitar la prestación de servicios turísticos relacionados con el cultivo de las berries y su gastronomía; esto puede suponer unos ingresos importantes, así como la difusión de una buena imagen de estos productos en mercados muy exigentes. Este tipo de beneficios afectaría en mayor medida a los grandes productores, por lo que se sobreentiende que son ellos los que deben realizar un mayor esfuerzo económico y reformador, mientras que los pequeños y medianos productores deberían llevar a cabo un conjunto de medidas mínimas que, sin mucho esfuerzo económico, les permitan dar un aspecto suficientemente armónico a estas explotaciones. 


\section{Bibliografía}

Consejo de Europa, 2000. Convenio Europeo del Paisaje, Florencia 20/10/2000. [En línea, febrero 2019]: https://www.boe.es/buscar/doc.php?id=BOE-A-2008-1899

Guzmán Casado, G.I.; Alonso Mielgo, A.M.; 2008. Buenas prácticas en la producción ecológica: Funcionalidad de los setos. Ministerio de Medio Ambiente, Medio Rural y Marino. Madrid.

Junta de Andalucía, 2012. Estrategia de Paisaje de Andalucía. Consejería de Medio Ambiente y Ordenación del Territorio. Sevilla http://www.juntadeandalucia.es/medio ambiente/portal_web/web/temas_ambientales/evaluacion_integracion_planificacion/pl anificacion_ambiental/estrategias/estrategia_paisaje/Estrategia_de_Paisaje_de_Andalu cia_2012.pdf

Junta de Andalucía, 2017. Guía para la aplicación de las medidas medioambientales OPFH a partir de 1 de junio de 2017. Consejería de Agricultura, Pesca y Desarrollo Rural. Dirección General de Ayudas Directas y de Mercados. [En línea, octubre 2018]: https: //www.juntadeandalucia.es/export/drupaljda/estudio_adaptacion_medidas_medioambi entales_v4.pdf

Junta de Andalucía, 2018. Consejería de Agricultura, Pesca y Desarrollo Rural. SIGPAC2018. [En línea, octubre 2018]: https://www.juntadeandalucia.es/organismos/agricultu rapescaydesarrollorural/areas/politica-agraria-comun/paginas/sigpac-descargainformacion-geografica-shapes-provincias.html

Sánchez Haro, R.; 2018. Declaraciones del Consejero de Agricultura, Pesca y Desarrollo Rural de la Junta de Andalucía recogidas por Huelva Buenas Noticias en la presentación del IV Congreso de Frutos Rojos. [En línea, octubre 2018]: https://huelvabuenasnoti cias.com/2018/02/07/los-frutos-rojos-de-huelva-generan-mas-de-920-millones-deeuros-en-ventas-al-exterior/

Swanwick, C.; and Land Use Consultants. 2002. Landscape Character Assessment. Guidance for England and Scotland. The Countryside Agency, Gloucestershire, and Scottish Natural Heritage, Edinburgh.

Villa, A.; y Giansur Consultores, 2008. Manual de Buenas Prácticas Agrarias Sostenibles de los Frutos Rojos. Fundación Doñana 21. Junta de Andalucía. Sevilla. [En línea, octubre 2018]: http://donana.es/source/MANUAL\%20FRUTOS\%20ROJOS.pdf

Zoido Naranjo, F.; (Dir.) 2014. Bases para la realización del Sistema Compartido de Información sobre el Paisaje de Andalucía. Consejería de Medio Ambiente y Ordenación del Territorio, Junta de Andalucía. Sevilla. 527 p.

Zoido Naranjo, F.; y Rodríguez Rodríguez, J.; (Dirs.) 2014. Sistema Compartido de Información del Paisaje de Andalucía. Aplicación al litoral. Secretaría General de Ordenación del Territorio (SGOTU). Consejería de Medio Ambiente y Ordenación del Territorio. Junta de Andalucía. Sevilla. 606 p. 
